Dans le cadre de l'exercice

de leur double mission

de recherche scientifique

d'une part, et de service à

la recherche et à l'industrie

d'autre part, les centres de

rayonnement synchrotron

sont en constante inter-

action avec les entreprises.

Ceci concerne aussi bien la

conception, la construction,

l'exploitation et la

maintenance des équipements

de production de lumière

synchrotron, que l'utilisation

de celle-ci dans les études

de la matière vivante et

des matériaux complexes

d'intérêt industriel ou sociétal.

\title{
Utilisation des lignes de lumière pour des problématiques industrielles
}

Philippe Deblay (1) (philippe.deblay@synchrotron-soleil.fr) et Edward P. Mitchell ${ }^{2)}$ (mitchell@esrf.fr) (1) Service Relations Industrielles \& Valorisation, Synchrotron SOLElL, L'Orme des Merisiers, BP 48, Saint-Aubin, 91192 Gif-sur-Yvette Cedex

(2) Business Development Office, European Synchrotron Radiation Facility, 6 rue Jules Horowitz, 38043 Grenoble Cedex 9

\section{Les secteurs industriels et les} types d'entreprises concernés par les applications synchrotron

Les lignes de lumière synchrotron sont des équipements de hautes performances qui permettent de mener des études multimodales et multiéchelles sur des matériaux d'intérêt industriel ; les entreprises industrielles et de service à l'industrie qui utilisent les installations de SOLEIL et de l'ESRF sont issues de secteurs très variés, mettant en œuvre la matière vivante ou les matériaux complexes : pharmacie, biotechnologies, cosmétiques, agroalimentaire, chimie, pétrochimie, matériaux (métaux, alliages, plastiques, céramiques, verres...), microélectronique, aérospatial, automobile, environnement, énergie...

La demande industrielle est globalement tirée par les applications en biologie/ santé : les secteurs de la pharmacie, des biotechnologies et des cosmétiques représentent chacun entre 21 et $26 \%$, et ensemble plus de $70 \%$ des prestations confidentielles réalisées depuis le début d'exploitation de SOLEIL ; environ $40 \%$ de l'activité commerciale de l'ESRF repose sur le seul secteur de la pharmacie [1], utilisateur de la cristallographie des protéines pour la découverte de nouveaux médicaments [2]. Cette demande industrielle a largement provoqué le développement de protocoles d'automatisation sur les lignes de lumière de biocristallographie, qui bénéficient également aux équipes académiques et sont maintenant appliqués à d'autres techniques [3].

Les entreprises clientes de SOLEIL et de l'ESRF, pour des prestations d'analyses synchrotron liées à des problématiques industrielles, sont de taille et de typologie variées, regroupées autour de quatre grandes familles :
- des Jeunes Entreprises Innovantes, principalement du secteur de la biotechnologie ou de celui des "technologies vertes ", qui sont le plus souvent issues de la recherche publique et qui ont généralement au moins une première expérience de l'utilisation de lignes de lumière synchrotron ;

- des PME à la recherche d'équipements d'analyse, de caractérisation ou de contrôle performants, permettant de répondre à des besoins ponctuels, mais urgents, tels qu'une pollution sur une chaîne de fabrication ou la compréhension du comportement d'un produit en cours de vieillissement ;

- des centres de recherches de grands groupes internationaux qui mènent des activités variées de $\mathrm{R} \& \mathrm{D}$, de $\mathrm{R} \& \mathrm{~T}$ et d'innovation, et dont les équipes utilisent le plus souvent de manière fréquente, voire récurrente, les techniques synchrotron ;

- des EPIC (Établissements Publics à caractère Industriel et Commercial) travaillant sur des problématiques industrielles génériques pour l'ensemble d'une filière industrielle, par exemple dans les domaines de l'aérospatial, de l'énergie, de la pétrochimie ou des mines.

\section{Les besoins industriels et les solutions synchrotron}

Contrairement à une idée reçue persistante, les besoins industriels en analyses de matériaux par des techniques synchrotron ne sont pas limités aux seules activités de $\mathrm{R} \& \mathrm{D}$ pour la mise au point ou la caractérisation de nouveaux produits à faible niveau de maturité technologique ; ils concernent également d'autres phases du cycle de vie des produits, tels que le contrôle qualité ou le suivi de fabrication, ou encore le vieillissement, le recyclage 
>>

et la valorisation en fin de vie. Les analyses synchrotron sont également utilisées dans le cadre d'expertises judiciaires, par exemple dans le domaine de l'environnement pour régler des litiges de pollution entre entreprises géographiquement proches.

L'ESRF et SOLEIL proposent et réalisent des prestations d'analyse synchrotron sur mesure, en fonction des besoins spécifiques de chaque projet proposé par chaque client. Ces analyses permettent d'accéder aux caractéristiques et aux propriétés les plus intimes du matériau étudié, chaque technique synchrotron donnant des informations chimiques, structurales, morphologiques ou électroniques, voire magnétiques.

Tout demandeur industriel choisit l'une des trois modalités d'accès aux équipements et aux compétences synchrotron : projet scientifique publiable, prestation payante confidentielle, partenariat de $\mathrm{R} \& \mathrm{D}$ avec les équipes de ligne (voir l'article «Les modalités d'accès à l'ESRF et à SOLEIL », p. 108).

Dans le cas d'une prestation payante confidentielle, les deux synchrotrons peuvent intervenir de manière graduée, en fonction notamment des compétences synchrotron et de la disponibilité du client :

- Mise à disposition d'une ligne de lumière pour permettre au client et/ou à un prestataire de $\mathrm{R} \& \mathrm{D}$ de son choix (laboratoire de recherche publique ou entreprise de services d'analyse) de collecter les données expérimentales, avec l'assistance du personnel de ligne.

- Mise à disposition d'une ligne de lumière, avec intervention directe du personnel du synchrotron pour :

- la préparation éventuelle des échantillons avant passage sur la ligne de lumière ;

- la collecte des données expérimentales par le personnel de ligne, en présence ou non du client et/ou du prestataire de R\&D de son choix, l'analyse et le traitement des données expérimentales étant réalisés par la suite par le client (ou par son prestataire).

- Prestation d'étude complète «de l'échantillon à l'échantillon avec son rapport d'analyse ", avec prise en charge intégrale de toutes les étapes : réception des échantillons, collecte des données expérimentales sur la ligne de lumière, analyse et traitement de ces données expérimentales, remise des échantillons analysés et d'un rapport de synthèse des résultats.

SOLEIL et l'ESRF collaborent également avec l'industrie par l'intermédiaire d'étudiants en thèse ou de postdoctorants, financés directement par l'industrie ou par les acteurs publics (ANR, programmes européens...). Dans le cadre d'un programme commun de recherche, la contribution du synchrotron s'effectue classiquement en temps de faisceau et en expertise liée à la mise en œuvre des équipements synchrotron, l'apport de l'industriel étant généralement réalisé par son expertise des problématiques spécifiques de son secteur d'activité. Ce type de partenariat gagnant-gagnant est un moyen très efficace de rapprocher la recherche et l'industrie, non seulement en générant des résultats d'intérêt industriel, mais aussi en formant des scientifiques qualifiés capables d'assurer une interface efficace entre les entreprises et les environnements synchrotron.

\section{Des applications dans des secteurs industriels variés}

\section{Exemple 1 : Analyse de la précipitation dans des aciers ODS renforcés par dispersion d'oxydes [4]}

Les aciers ferritomartensitiques renforcés par dispersion d'oxydes nanométriques (nanoparticules de taille inférieure à $3 \mathrm{~nm})$ d'yttrium et de titane, dits ODS (Oxide Dispersion Strengthened), sont de bons candidats pour différents composants des centrales nucléaires de $4^{\text {e }}$ génération (notamment la gaine du combustible), en raison de leur haute résistance à l'irradiation (absence de gonflement à forte dose d'irradiation) et de leurs propriétés mécaniques élevées à haute température (faible déformation de fluage).

Les alliages ODS sont élaborés principalement par "mécanosynthèse " (voie mécanique issue de la métallurgie des poudres). La matrice (poudre d'acier préalliée) est cobroyée avec 0,2 à 0,6\% en poids de poudre d'oxyde d'yttrium. Durant cette étape, l'oxyde se dissout ; puis un phénomène de nano-précipitation apparaît pendant la phase de consolidation, effectuée par extrusion ou compression isostatique à chaud.
Les expériences, effectuées sur un grain unique de poudre à base de $\mathrm{Fe}-9 \% \mathrm{Cr}$ et sur un échantillon consolidé à base de $\mathrm{Fe}-18 \% \mathrm{Cr}$, ont montré la capacité du rayonnement synchrotron à caractériser finement la dispersion de la phase oxyde des aciers ODS. Par ailleurs, elles ont permis d'étudier le mécanisme de précipitation dans différents aciers ODS non irradiés, élaborés au CEA.

La complémentarité des deux techniques utilisées (diffraction des rayons $\mathrm{X}$ et spectroscopie d'absorption X) sur la ligne MARS (Multi-Analyses on Radioactive Samples) de SOLEIL est particulièrement bien adaptée aux analyses structurales (fig. 1) et de l'environnement chimique local (fig. 2) de ces nanoparticules.

Le diffractogramme obtenu (fig. 1) sur un grain unique de poudre après recuit, montre la coexistence des deux formes cristallines de l'oxyde $\mathrm{Y}_{2} \mathrm{O}_{3}$ : cubique centrée (bcc) et monoclinique centrée (bcm). La grande brillance du rayonnement synchrotron, la haute résolution du monochromateur de la ligne ainsi que la haute sensibilité du détecteur ont permis de distinguer les raies caractéristiques de la structure bcm de $\mathrm{Y}_{2} \mathrm{O}_{3}$ dans le domaine d'angle de diffraction $2 \theta$ situé entre 13,7 et $15,7^{\circ}$, ce que ne permettait pas la diffraction des neutrons.

Les spectres EXAFS de la figure 2, obtenus au seuil d'absorption $\mathrm{K}$ de l'yttrium, montrent que le signal des nanoparticules dans l'alliage ODS à $9 \%$ de chrome contenant $10 \%$ d'oxyde d'yttrium, est très similaire à celui de l'oxyde de référence $\left(\mathrm{Y}_{2} \mathrm{O}_{3}-\mathrm{bcc}\right)$, y compris pour les atomes situés à grande distance.

En revanche, dans le cas de l'alliage ODS à $18 \%$ de chrome et $0,6 \%$ d'oxyde d'yttrium, les amplitudes et les positions des pics des transformées de Fourier du signal d'EXAFS de l'yttrium (qui représentent une pseudo-distribution de fonction radiale de l'enveloppe de coordination autour de l'atome absorbeur Y) sont significativement différentes. Ce résultat pourrait s'expliquer par la coexistence de structures autres que $\mathrm{Y}_{2} \mathrm{O}_{3}$ cubique centré, telle que celle de $\mathrm{Y}_{2} \mathrm{Ti}_{2} \mathrm{O}_{7}$, qui a déjà été identifiée par les mesures de diffraction $\mathrm{X}$ sur la ligne MARS. Ces oxydes de taille nanométrique ont été également vus en microscopie électronique en transmission et analysés par sonde atomique tomographique. 


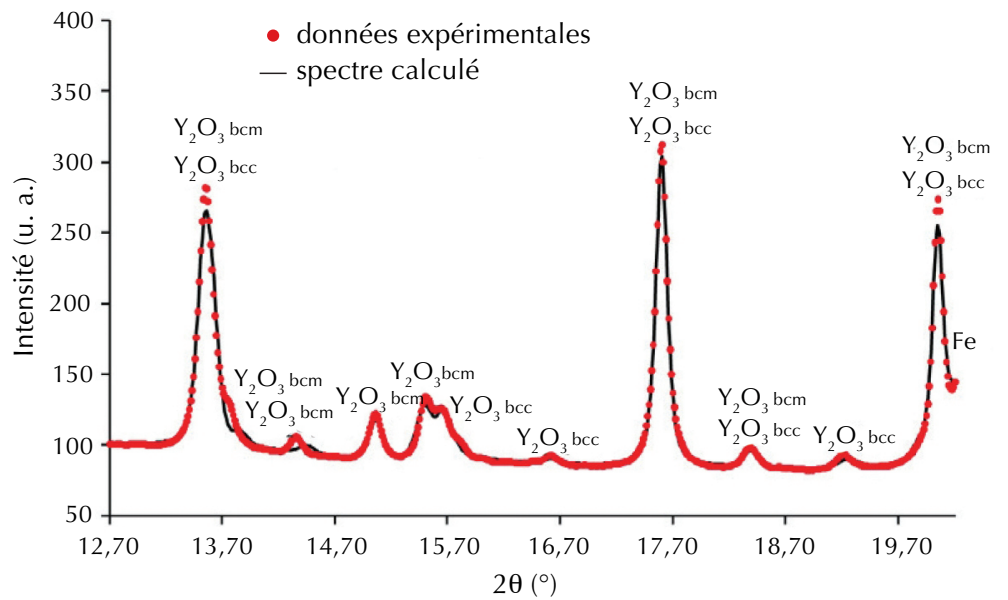

1. Spectre de diffraction X obtenu pour un grain unique de poudre d'alliage ODS à $9 \%$ de chrome, d'une dimension d'environ $50 \mu \mathrm{m}$, après mélange pendant 48 heures et recuit 1 heure à $1100^{\circ} \mathrm{C}$.

\section{Spectres EXAFS au seuil d'absorption $K$ de} I'yttrium, obtenus pour (i) l'alliage ODS à $18 \%$ de chrome, consolidé, puis recuit pendant 1 heure à $1300^{\circ} \mathrm{C}$ (courbe rouge) ; (ii) l'alliage 0DS à $9 \%$ de chrome, obtenu par mélange des poudres pendant 48 heures, puis recuit 1 heure à $1100^{\circ} \mathrm{C}$ (courbe verte) ; et (iii) le matériau de référence $\mathrm{Y}_{2} \mathrm{O}_{3}$-bcc (courbe bleue). En abscisse : le nombre d'onde $k$ du photoélectron émis lors du processus d'absorption ; en ordonnée : les oscillations $\chi(k)$ du coefficient d’absorption au-dessus du seuil, multipliées par $k^{2}$.
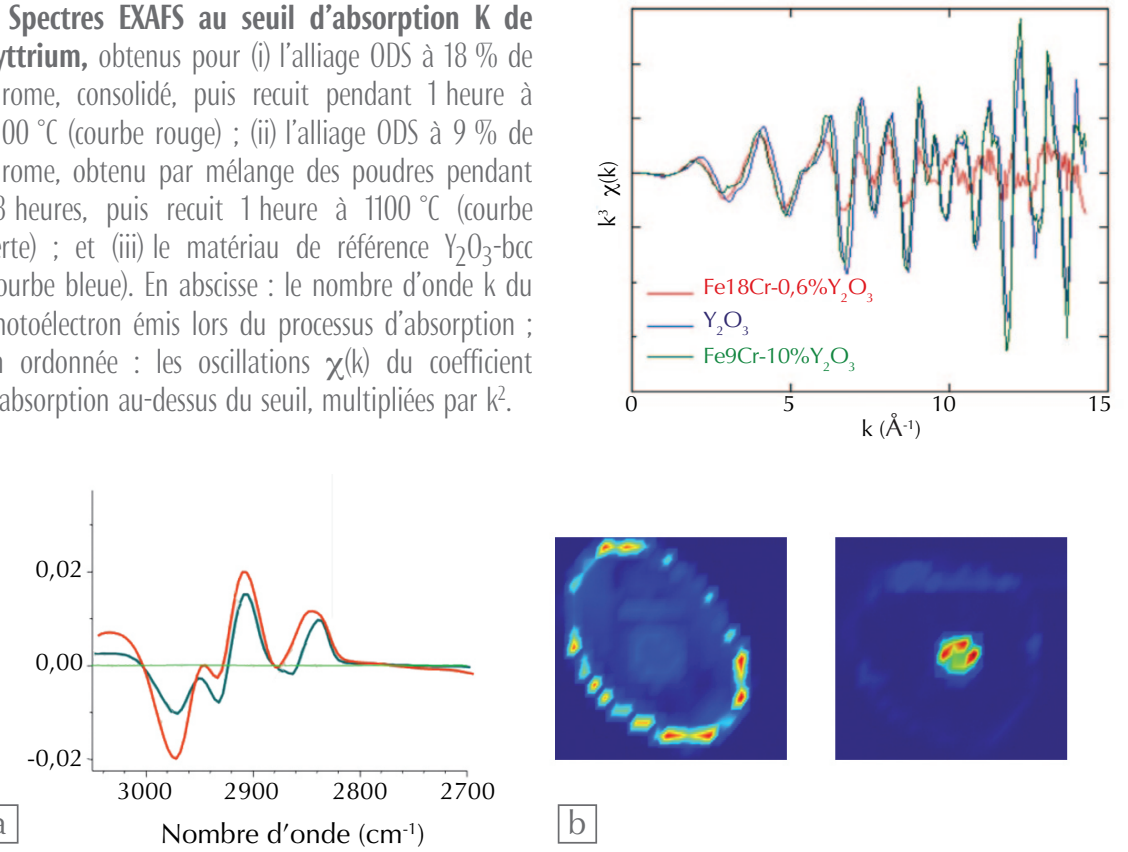

$\mathrm{b}$

3. (a) Mise en évidence de deux types de lipides dans la cuticule (courbe rouge) et le canal médullaire (courbe bleue) d'un cheveu humain, par microspectroscopie IR. (b) Visualisation des lipides dans la cuticule (à gauche) et dans le canal médullaire (à droite). (Expériences réalisées sur la ligne SMIS du synchrotron SOLEIL.)

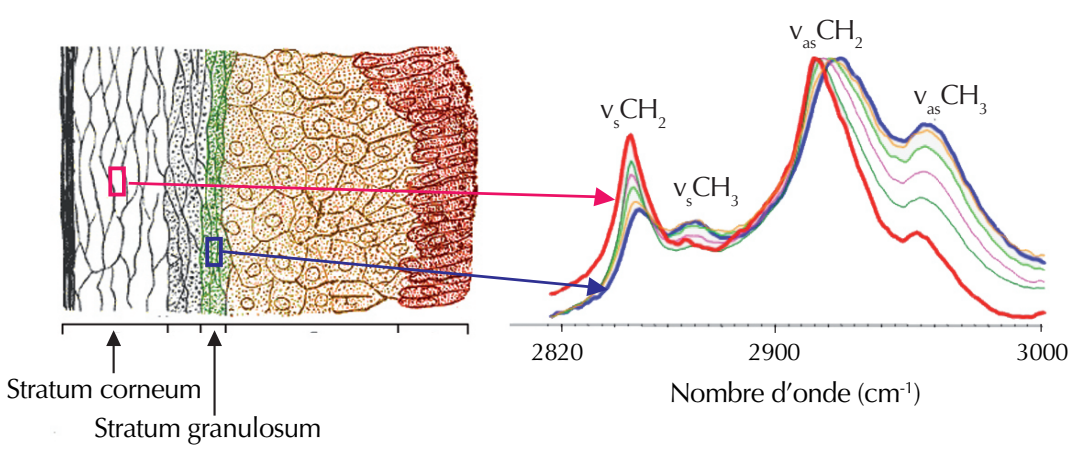

4. Mise en évidence, par spectroscopie infrarouge, des lipides dans le stratum corneum (courbe rouge) et le stratum granulosum (courbe bleue) de l'épiderme de peau humaine. (Expériences réalisées sur la ligne SMIS du synchrotron SOLEIL.) À gauche : coupe de l'épiderme, montrant les deux régions étudiées.
Exemple 2 : Micro-imagerie infrarouge synchrotron pour le secteur de la cosmétique

La lumière synchrotron infrarouge est utilisée dans le secteur de la cosmétique pour déterminer la composition chimique des différents compartiments du cheveu et de la peau, avec des résolutions spatiales de l'ordre de quelques micromètres. La cuticule pour les cheveux [5] et le stratum corneum pour la peau [6], ont fait l'objet d'études spécifiques sur la ligne SMIS de SOLEIL pour comprendre le rôle des couches lipidiques dans la protection de ces tissus humains. Lors des traitements cosmétiques, le double rôle de pénétration de l'agent actif dans les couches sous-jacentes (respectivement cortex pour le cheveu et épiderme pour la peau) et de préservation de la structure de ces couches de protection lipidiques a été validé et caractérisé (figs 3 et 4).

La figure 3 met en évidence, par l'analyse des signatures spectroscopiques dans la région spécifique entre 2800 et $3000 \mathrm{~cm}^{-1}$, la nature différente des lipides constitutifs de deux régions caractéristiques du cheveu : la cuticule, enveloppe externe d'environ $5 \mu \mathrm{m}$ d'épaisseur, et le canal médullaire, canal central ayant un diamètre de 15 à $20 \mu \mathrm{m}$. La localisation différentielle des lipides dans ces deux régions, visualisée par les images chimiques (fig. 3b), a été obtenue grâce à la haute résolution spatiale $(3 \mu \mathrm{m} \times 3 \mu \mathrm{m}$ sur la ligne de microimagerie infrarouge SMIS de SOLEIL) que permet la brillance particulièrement importante de la source synchrotron.

La figure 4 montre que, dans la même région spectrale que celle utilisée pour l'étude du cheveu (entre 2800 et $3000 \mathrm{~cm}^{-1}$ ), les positions des fréquences correspondant aux longues chaines alkyles $\left(-\mathrm{CH}_{2}\right)$ des lipides se déplacent en fonction de la profondeur de l'épiderme, du stratum corneum, couche cornée la plus externe de l'épiderme, d'environ 10 à $40 \mu \mathrm{m}$ d'épaisseur (5 à 20 assises cellulaires, selon la région du corps), jusqu'au stratum granulosum, couche granuleuse plus profonde ne contenant que trois à quatre assises de cellules. Ce déplacement dans le stratum corneum résulte de la structuration orthorhombique des chaînes lipidiques, et peut être interprété comme une désorganisation de cette couche de l'épiderme, liée au phénomène continu de renouvellement cellulaire. 
$>>$

Exemple 3 : Tomographie $X$ à haute résolution pour l'optimisation énergétique de la production de verres

La tomographie $\mathrm{X}$ à haute résolution permet de visualiser de manière non destructive la micro-organisation interne d'une large gamme de matériaux [7], offrant à cette technique de vastes applications dans de nombreux secteurs industriels. Le CNRS et le groupe Saint-Gobain étudient, par exemple, des mélanges de sable de silice et de carbonates de sodium et de calcium, de même nature que ceux utilisés pour la fabrication des verres à vitres [8]. Ce mélange est porté à $1500{ }^{\circ} \mathrm{C}$ pendant plusieurs jours dans les fours industriels, générant une forte consommation d'énergie. Comprendre les différentes étapes de la transformation des matières premières, ainsi que le couplage entre les réactions chimiques et la microstructure du mélange initial, est nécessaire pour réduire cette consommation énergétique.

Une observation de la réaction à haute température, in situ et en temps réel, a été réalisée pour la première fois par tomographie $\mathrm{X}$ sur la ligne de lumière ID15A de l'ESRF ; elle a donné toutes les quinze secondes des images $3 \mathrm{D}$ de la réaction en cours, avec une résolution spatiale de 1,6 $\mu \mathrm{m}$. Ces images uniques révèlent l'importance des contacts entre grains d'espèces différentes (fig. 5). Selon l'absence ou la présence de tels contacts, le carbonate peut en effet, soit être incorporé à un liquide très réactif, soit produire des défauts cristallins.

\section{Renforcement de l'utilisation industrielle des synchrotrons par l'hébergement de projets}

Au-delà de l'utilisation "ponctuelle » des lignes de lumière pour des projets à court terme de recherches académique ou industrielle, SOLEIL et l'ESRF se sont engagés dans une démarche d'hébergement de projets à moyen ou long terme au sein de plates-formes de recherche et de services, spécialisées par domaine applicatif.

Les caractéristiques de ces plates-formes sont les suivantes :

- installation sur le site du synchrotron, dans des locaux dédiés ;

- activité d'hébergement d'équipes externes, publiques et/ou privées, sur des périodes longues (de quelques mois à quelques années) ;

- mise à disposition d'une structure d'interface $\mathrm{R} \& \mathrm{D}$ et de support, dotée de moyens humains propres, permettant de traduire les problématiques spécifiques du domaine applicatif en besoins de solutions synchrotron;

- accès aux équipements synchrotron et à des équipements complémentaires non synchrotron, nécessaires aux problématiques spécifiques du domaine applicatif.

Un premier exemple de réalisation à SOLEIL est la plate-forme IPANEMA, dans le domaine des matériaux anciens. Deux autres projets de plates-formes spécialisées viennent d'être lancés, début 2013, par SOLEIL et ses partenaires :
- la plate-forme mutualisée d'innovation Cosmétomique, pour les entreprises françaises du secteur de la cosmétique, dédiée à la caractérisation et à la mesure de l'efficacité et de l'innocuité des produits, dans le cadre d'un partenariat avec la PME BIO-EC (91160 Longjumeau) et l'Université de CergyPontoise ;

- la plate-forme MICASOL, portée par les laboratoires matériaux du CNRS et des deux universités de la Région Alsace, en liaison avec l'Institut Carnot MICA, et destinée aux entreprises alsaciennes de tous secteurs intéressées par la caractérisation des matériaux, de l'échelle moléculaire à l'échelle macroscopique.

L'industrie de la micro et nanoélectronique, présente dans la région grenobloise, est confrontée à différents problèmes de matériaux, tels que la connaissance de la structure et de la taille des grains, la détermination des champs de contraintes résiduelles dans les cristaux, la diffusion intermétallique ou encore les mécanismes de défaillance. Afin de soutenir le développement technologique et commercial de cette industrie à forte implication locale, un projet est en cours de structuration, pour permettre un accès facilité aux installations de l'ESRF et à celles du centre de diffusion neutronique de l'Institut Laue Langevin (ILL). Ce projet de rapprochement des grandes installations grenobloises et de la R\&D industrielle du secteur de la micro et nanoélectronique prévoit, notamment, un financement de 6,5 M€ pour l'Institut

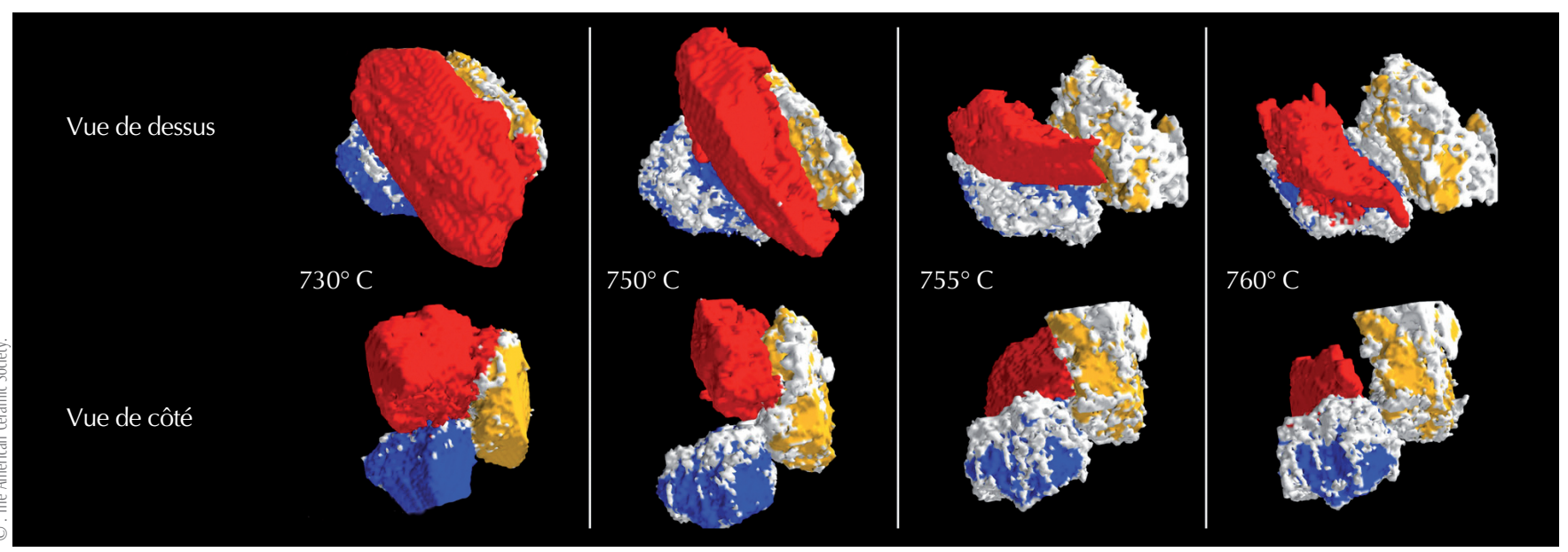

5. Réactions entre un grain de carbonate de sodium (rouge) et deux grains de sable de silice (bleu et jaune), à différentes températures et observées sous deux angles différents. Ces réactions produisent des silicates de sodium (blanc), précurseurs du verre. Les mouvements du grain de carbonate de sodium, qui s’accroche successivement à l'un de ses voisins, puis à l'autre, favorisent l'avancement de la réaction. La taille des grains de sable est d'environ 100 microns. 
de recherche technologique Nanoelec, permettant la mise en place de démonstrateurs de nanodispositifs et de procédés de mesure et de test utilisant les rayons $\mathrm{X}$ synchrotron et les neutrons.

\section{Conclusion}

Le développement de l'utilisation des lignes de lumière par les industriels représente un double enjeu pour SOLEIL et pour l'ESRF : d'une part, contribuer au maintien, voire au renforcement de la compétitivité des entreprises françaises et européennes utilisatrices, qui disposent alors d'avantages concurrentiels décisifs pour la mise au point et le contrôle de leurs produits; et, d'autre part, assurer une meilleure rentabilité économique des investissements réalisés pour construire et exploiter les installations synchrotron.

Dans un contexte européen, l'ESRF et SOLEIL sont partenaires dans le projet CALIPSO $^{(1)}$, financé par le $7^{\text {e }}$ PCRDT et comprenant un ensemble de travaux spécifiques pour catalyser les relations avec l'industrie.

Cette synergie entre recherche scientifique et innovation industrielle, mise en place de manière volontariste à l'ESRF et à SOLEIL, correspond aux objectifs de la stratégie Europe 2020, dont le programmecadre HORIZON 2020 sera conçu de manière à mieux intégrer la recherche et l'innovation autour de trois priorités : l'excellence scientifique, la primauté industrielle et les défis sociétaux.

(1) CALIPSO : Coordinated Access to LIght sources to Promote Standards and Optimization.

\section{Références}

• E.P. Mitchell et al., Synchrotron Radiation News, 24 (2011) 18-23.

2-M.W. Bowler et D. Nurizzo, voir p. 48.

3• S. Malbet-Monaco et al., Acta Crystallographica D, sous presse.

4 J.L. Béchade et al., Journal of Nuclear Materials, 428 (2012) 183-191.

5• L. Kreplak et al., International Journal of Cosmetic Science, 23 (2001) 369-374.

6• N. Gross et al., IFSCC Magazine, 7 (2004) 1-6.

7• J. Baruchel et al., voir p. 32.

8• E. Gouillart et al., J. Am. Ceram. Soc., 95 (2012) 1504-1507. 